\title{
La Mitigación de heladas en los camellones del altiplano andino
}

L'atténuation des gelées dans les ados de l'altiplano andin

Frost Mitigation in the raised fields of the andean altiplano

Jean-Paul Lhomme y Jean-Joinville Vacher

\section{CpenEdition}

Journals

Edición electrónica

URL: https://journals.openedition.org/bifea/6556

DOI: $10.4000 /$ bifea. 6556

ISSN: 2076-5827

Editor

Institut Français d'Études Andines

Edición impresa

Fecha de publicación: 1 septiembre 2003

Paginación: 377-399

ISSN: 0303-7495

Referencia electrónica

Jean-Paul Lhomme y Jean-Joinville Vacher, «La Mitigación de heladas en los camellones del altiplano andino», Bulletin de l'Institut français d'études andines [En línea], 32 (2) | 2003, Publicado el 08 agosto 2003, consultado el 07 diciembre 2022. URL: http://journals.openedition.org/bifea/6556 ; DOI: https:// doi.org/10.4000/bifea.6556

\section{(ब) $\Theta \Theta$}

Creative Commons - Atribución-NoComercial-SinDerivadas 4.0 Internacional - CC BY-NC-ND 4.0 https://creativecommons.org/licenses/by-nc-nd/4.0/ 


\title{
LA MITIGACIÓN DE HELADAS EN LOS CAMELLONES DEL ALTIPLANO ANDINO
}

\author{
Jean-Paul LHOMME*a, Jean Joinville VACHER ${ }^{* * a}$
}

\section{Resumen}

El sistema de camellones o "waru warus" es una antigua técnica agrícola de manejo del suelo y del agua. En los tiempos prehispánicos era muy frecuente en la región del lago Titicaca. Consiste esencialmente en una serie de plataformas de tierra rodeadas por canales de agua. Las plantas se cultivan sobre las plataformas y el nivel del agua en los canales puede controlarse a través de entradas y salidas de agua. Un beneficio importante y ampliamente reconocido de este sistema de manejo en el altiplano es su contribución a la mitigación de heladas nocturnas durante la campaña agrícola. Con el objetivo de cuantificar este fenómeno y describir los procesos físicos responsables de la mitigación, se ha realizado un experimento en la región del lago Titicaca sobre un sistema de camellones cultivado con papas comparándolo con una parcela "testigo" en la "Pampa". Se presentan resultados experimentales que evidencian por una parte, el valor elevado de la temperatura del agua con respecto a la del cultivo sobre las plataformas, y por otra, una temperatura de cultivo siempre mayor (1-2 grados) en los camellones que en la Pampa. Conjuntamente se presenta un modelo mecanístico adaptado de un esquema de transferencia bicapa de tipo "Shuttleworth-Wallace" (una capa de vegetación y un sustrato de agua). El modelo precisa el papel que juegan los canales en la dinámica del calor y por lo tanto en la variación de la temperatura del cultivo durante la noche. El efecto de mitigación se debe al flujo de calor que emana del agua y a menudo también a la condensación del vapor de agua sobre las hojas del cultivo. Utilizando el modelo de manera predictiva, se muestra que canales más anchos o plataformas más estrechas tienen un impacto positivo sobre la temperatura mínima del cultivo alcanzada durante la noche. Aumentar la profundidad del agua mejora también la mitigación de heladas, pero a la inversa, un canal más profundo (con el mismo nivel de agua) tiene un impacto negativo. Aumentar el índice de área foliar (LAI) o la altura del cultivo tiene un efecto positivo sobre la mitigación de heladas (el beneficio marginal, sin embargo, es muy pequeño cuando el índice foliar supera el valor 1). Mayor velocidad de viento o mayor humedad relativa incrementa también el efecto de mitigación de heladas.

Palabras claves: Camellones, altiplano andino, mitigación de heladas, modelo, dinámica del calor, temperatura del cultivo.

*Dirección de Agrometeorología, Servicio Nacional de Meteorología e Hidrología(SENAMHI), Lima, Perú. E-mail: 1homme@amauta.rcp.net.pe

${ }^{* *}$ Institut français d'Études andines, Av. Arequipa 4595, Miraflores Lima, Perú. E-mail: jvacher@ifea.org.pe

${ }^{a}$ Dirección permanente: IRD, UR060, 213 rue La Fayette, 75010 Paris, France. 


\section{L'ATTÉNUATION DES GELÉES DANS LES ADOS DE L'ALTIPLANO ANDIN Résumé}

Le système des ados ou waru warus, est une ancienne technique agricole qui était très fréquente dans la région du lac Titicaca à l'époque préhispanique. Elle consiste essentiellement en une série de plateformes de terre surélevées entourées par des canaux. Les plantes se cultivent sur les plateformes et le niveau d'eau dans les canaux est contrôlé par les entrées et sorties. Un bénéfice important et largement reconnu de ce système de culture sur l'altiplano est sa contribution à l'atténuation des gelées nocturnes durant la campagne agricole. Dans le but de quantifier ce phénomène et de décrire les processus physiques responsables de cette atténuation une expérimentation a été conduite dans la région du lac Titicaca en comparant un système d'ados cultivés avec des pommes de terre avec une parcelle témoin dans la "Pampa". Les résultats expérimentaux mettent en évidence, d'une part la valeur élevée de la température de l'eau par rapport à celle de la culture sur les plateformes, d'autre part une température de la culture toujours plus élevée (1-2 degrés) dans les ados que dans la Pampa. On présente également un modèle mécaniste, adapté d'un schéma de transfert bi-couche de type "Shuttleworth-Wallace" (une couche représentant la végétation et une couche représentant le substrat d'eau). Le modèle précise le rôle joué la nuit par les canaux dans la dynamique de la chaleur et dans la variation de la température de la culture. Il apparaît que l'effet d'atténuation est dû principalement au flux de chaleur sensible qui émane de l'eau, mais souvent aussi à la condensation de vapeur d'eau sur les feuilles de la culture. En utilisant le modèle de manière prédictive on montre que des canaux plus larges ou des plateformes plus étroites ont un impact positif sur la température minimale de la culture atteinte durant la nuit. Augmenter la profondeur de l'eau améliore également l'effet d'atténuation des gelées, mais à l'inverse, un canal plus profond, avec le même niveau d'eau, a un impact négatif. L'augmentation de la surface foliaire (LAI) ou de la hauteur du couvert a un effet positif sur l'atténuation des gelées. Une vitesse de vent plus forte ou une humidité relative de l'air plus élevée accroît également l'effet d'atténuation.

Mots clés : Ados, altiplano andin, atténuation des gelées, modèle, dynamique de la chaleur, température de culture.

\section{FROST MITIGATION IN THE RAISED FIELDS OF THE ANDEAN ALTIPLANO}

\section{Abstract}

The raised fields system is an old technique of soil and water management, which was very frequent in the Lake Titicaca region before the Spanish conquest. It essentially consists of a series of earth platforms surrounded by water canals. Crops are grown on the platforms and water level in the canals is controled through inlet and outlet ditches. An important and widely recognized benefit of this system of management is its contribution to frost mitigation during the growing season. An experiment has been conducted on a system of raised fields in the Lake Titicaca region with the goal of quantifying the phenomenon and specifying the physical processes responsible for the mitigation effect. A potato crop was grown on the platforms of the raised fields and the same crop was grown on a control plot in the "Pampa". Experimental results show that during the night: (i) water temperature is much greater than the temperature of the crop on the platforms; (ii) crop temperature is always greater (1-2 degrees) on the platforms than on the control plot in the Pampa. A physical process-based model, adapted from a two-layer transfer scheme of the Shuttleworth-Wallace type (a vegetation layer and a substrate layer of water), is presented to explain the mitigation effect. The model specifies the role played by the canals in the nocturnal heat dynamics and the night-time variation of crop temperature. The mitigation effect is due to heat emanating from the canals and to water condensation on the leaves. When used in a predictive way, the model shows that wider canals and narrower platforms have a positive impact on the minimum crop temperature reached during the night. Increasing water depth also 
improves frost mitigation, but conversely, a deeper canal (with the same level of water) has a negative impact. Leaf area index (LAI) and crop height, both have a positive impact on frost mitigation. The marginal benefit, however, is very small when LAI is greater than 1 . Higher wind velocity or higher air relative humidity also enhances the frost mitigation effect.

Key words: Andean riased field, altiplano, frost mitigation, modelling, heat dynamic, crop temperature.

\section{INTRODUCCIÓN}

La agricultura en el altiplano de Bolivia y Perú se enfrenta a muy severas condiciones climáticas. Ubicada a más de $3800 \mathrm{~m}$ sobre el nivel del mar, esta región se caracteriza por sequías, heladas y granizadas frecuentes en el transcurso del crecimiento de los cultivos, de octubre a abril. Las poblaciones nativas, sin embargo, fueron capaces de desarrollar una agricultura próspera gracias a técnicas específicas adaptadas a las condiciones locales (tales como los andenes), en complemento a un control vertical de un máximo de pisos ecológicos (Murra, 1975) y al uso de la biodiversidad de cultivos andinos (Vacher, 1998). Dentro de estas técnicas existe un sistema particular de manejo del suelo y del agua, llamado "camellones" o "campos levantados" ("raised fields" en inglés y "ados" en francés). En Quechua se lo denomina "waru waru” y en Aymara "suka kollo". Estos camellones, la infraestructura agrícola más antigua en América del Sur (Erickson, 2000), consisten en una serie de plataformas de tierra rodeadas por canales de agua y ordenadas en haces de hileras paralelas entre sí. Se construyen cavando canales y redistribuyendo la tierra en forma de plataformas sobre-elevadas con respecto a la superficie original del suelo (Fig. 1). Los cultivos se instalan sobre las plataformas de tierra y los canales circundantes están conectados con entradas y

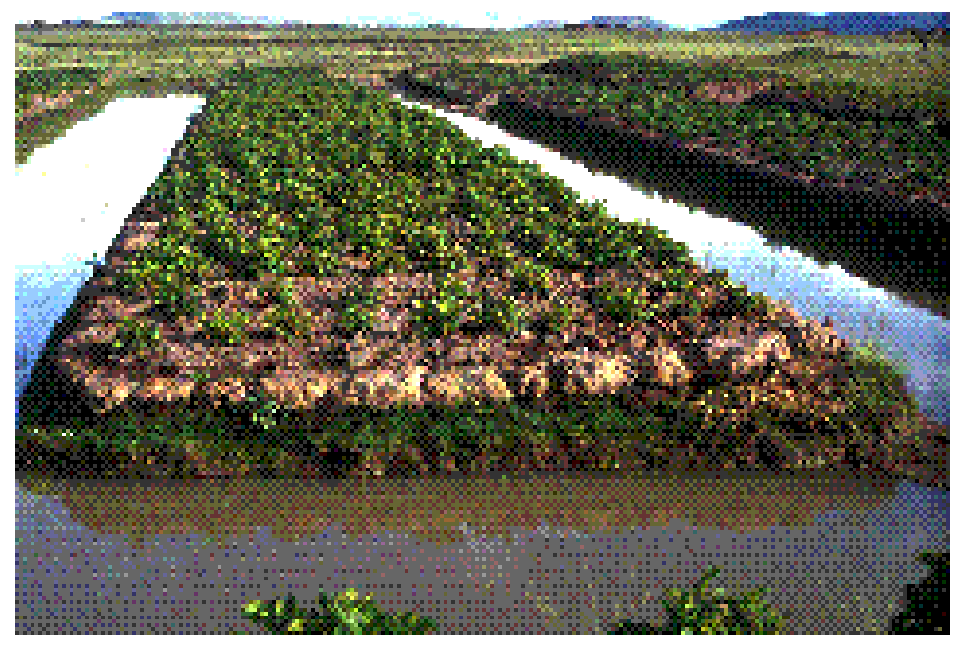

Fig. 1 - Vista de camellones en la región del Lago Titicaca. 
salidas de agua (Canahua \& Larico, 1992). La mayor extensión de antiguos camellones se encuentra en la región del lago Titicaca, con más de 100000 ha (Díaz \& Velázquez, 1992) a una distancia no mayor de $30 \mathrm{~km}$ de sus riberas, frecuentemente limitada a zonas bajas con riesgo de inundación. Hubo diferentes épocas de construcción, desde el primer milenio $\mathrm{AC}$, con diferentes épocas de abandono, posiblemente relacionadas con factores políticos y climáticos (Erickson, 1988; Bouysse et al., 1992). En la región de Tiahuanacu, por ejemplo, los camellones fueron abandonados alrededor de 1000 años DC, después de haber hecho posible el establecimiento de una densa población. En los últimos años, sin embargo, se ha desarrollado programas para la re-introducción y reconstrucción de estos sistemas de camellones, tales como el Programa Interinstitucional de Waru Waru (PIWA) bajo los auspicios del Proyecto Especial Binacional Lago Titicaca (PELT) (Canahua \& Larico, 1992). Se ha comprobado que esta rehabilitación no necesita cuantiosas inversiones.

Los camellones servían y sirven aún para cultivos tradicionales: tubérculos como la papa, la oca (Oxalis tuberosa) y granos como la quinoa (Chenopodium quinoa) y la cañihua (Chenopodium pallidicaule). Varias clasificaciones han sido propuestas para describir los sistemas de camallones. El programa PIWA los clasifica según la fuente de alimentación de agua en: fluviales, pluviales, lacustres, freáticos y mixtos (Cari \& Camacho, 1992). Smith et al. (1968) proponen una clasificación basada en la morfología de los camellones: damero (haces de surcos formando ángulos rectos con los adyacentes), patrón represado (grupos de camellones rodeados por bajas represas), patrón linear (camellones largos paralelos entre sí), patrón escalera (con plataformas en figuras alargadas en forma de escalera), patrón peinado (los camellones convergen hasta cegar los surcos intercalados dando la impresión de una horquilla o peine). Cabe señalar también en esta clasificación un sistema particular de cultivo que se asemeja a los camellones: el sistema de cultivo en laguna o qocha. Se encuentra esencialmente en el altiplano del sur del Perú, en el departamento de Puno: son áreas de cultivo en forma de depresiones que almacenan el agua de las lluvias y se secan en el invierno. Tienen dos diseños básicos, circular o rectangular, y las aguas se manejan por un canal de desagüe (Flores \& Paz, 1986).

La primera función de los camellones es evidentemente el control del agua y el aprovechamiento de las tierras pantanosas o inundables, como las que se encuentran cerca de lagos o ríos. No obstante, suministran otros beneficios tales como el riego durante períodos de sequía (superficial por acción manual del hombre o sub-superficial por infiltración lateral pasiva en el suelo a partir de los canales), la mitigación de heladas durante noches con enfriamiento radiativo y la producción de abono natural (Erickson, 1986a; 1986b; Grace, 1988). Para Smith et al. (1968) no es tan claro si fueron construidos para lograr el drenaje o la conservación del agua con vista a la irrigación o la mitigación de heladas. La conservación del agua es importante para poder regar en caso de déficit de lluvia y sembrar en la época más propicia, ya que los riesgos de sequía son muy altos en la región, así como los de heladas que destruyen los cultivos durante la estación de crecimiento. Si el proceso de riego superficial en los camellones por gravedad o por capilaridad es evidente, el mecanismo físico responsable del efecto de mitigación de heladas no es tan claro. Al menos tres mecanismos han sido avanzados para explicar la mitigación de heladas. Kolata \& Ortloff (1989) sugieren que la causa 
principal es la transferencia de calor por conducción en el suelo de los canales hacia las plataformas. Algunos autores (Riley \& Freimuth, 1979; Morlon, 1992) consideran que es el drenaje de aire frío de las plataformas hacia los canales que constituye el proceso físico principal responsable de la mitigación de heladas:

"La diferencia de niveles (altura) entre camellones y canales permite que las masas de aire frío, por su mayor densidad y peso, se deslicen del terraplén al canal" (Cari \& Camacho, 1992).

Para este propósito, cabe señalar sin embargo que la presencia de agua en los canales no es necesaria. No obstante la mayoría de los autores acuerdan considerar que la mitigación de heladas es principalmente la consecuencia del calor liberado por el agua en el aire (Smith et al., 1968; Erickson, 1986a; Morris, 1999). El agua de los canales actuaría como un amortiguador térmico, que evita o atenúa los efectos de las heladas. La cantidad importante de calor almacenada dentro de los canales durante el día, debido al calor específico elevado del agua y la intensa radiación solar, sería responsable de este efecto. El reciente y detallado estudio experimental de la dinámica del calor en los camellones por Sánchez de Lozada et al. (1998) confirma este hipótesis. Muestra claramente que el efecto de mitigación de heladas es esencialmente la consecuencia de procesos aéreos relacionados con la circulación del aire durante la noche de los canales hacia las plataformas.

El objetivo principal del presente estudio es el análisis de los procesos físicos responsables de la mitigación de las heladas radiativas en el sistema de camellones. Se presentan a la vez datos experimentales que complementan los de Sánchez de Lozada et al. (1998) y un modelo mecanístico que simula las transferencias de calor sensible y de vapor de agua (calor latente) dentro del sistema. En este sentido, el estudio constituye una extensión directa de la investigación reportada en el documento citado. El artículo consta de tres secciones principales. La primera sección presenta el experimento y analiza los datos obtenidos. La segunda detalla el modelo micrometeorológico y compara los datos simulados con los datos medidos. La tercera parte es dedicada a las predicciones del modelo, es decir, al efecto sobre la mitigación de heladas de la geometría de los camellones, de las características del cultivo y de las condiciones meteorológicas.

\section{ESTUDIO EXPERIMENTAL}

\section{1. Condiciones experimentales}

Experimentos micrometeorológicos sobre camellones tuvieron lugar en 1994 y 1995 en el altiplano boliviano cerca del lago Titicaca. Los campos experimentales se ubicaban sobre un terreno relativamente plano, perteneciente a CORDEPAZ (Corporación del Desarrollo de La Paz), en Kallutaca (16 ${ }^{\circ} 30^{\prime}$ de latitud, 68 $18^{\prime}$ de longitud), a aproximadamente $40 \mathrm{~km}$ de La Paz y $15 \mathrm{~km}$ de la orilla del lago, y a una altura de aproximadamente $3850 \mathrm{~m}$ sobre el nivel del mar. La precipitación media annual es de $590 \mathrm{~mm}, 80 \%$ de la lluvia cayendo durante la temporada de cultivo, de noviembre a abril. Las heladas pueden ocurrir casi todo el año. Los suelos son francoarcillosos a arcillosos. Alrededor de ocho hectáreas de camellones de tipo lineal-fluvial fueron construidos entre 1992 y 1994. La red de canales estaba conectada con el río Sini 
Jahuira, que servía para mantener agua en los canales, cuyo nivel era reajustado cada 3 ó 4 días. Una parcela de control, llamada parcela de la "Pampa", estaba ubicada a 300 $\mathrm{m}$ de las parcelas de camellones. A fin de tener las mismas condiciones meteorológicas sobre ambas parcelas, se evitó toda heterogeneidad topográfica entre los dos sitios. Los camellones, con una orientación Norte-Sur, tenían una longitud de $50 \mathrm{~m}$. El ancho de la plataforma $\left(w_{c}\right)$ era de $4 \mathrm{~m}$ y el del canal $\left(w_{w}\right)$ de $2 \mathrm{~m}$. La profundidad de los canales $\left(d_{c a}\right)$ era de 1,2 m, con una sección aproximadamente rectangular y con una profundidad mediana del agua $\left(d_{w}\right)$ de $0,8 \mathrm{~m}$. Los experimentos se realizaron al final de la campaña agrícola, durante los meses de marzo y abril, período durante el cual las heladas radiativas generan daños muy severos.

Al inicio de noviembre se sembró un cultivo de papa (Solanum tuberosum ssp andigena var Sani Imilla) en la parcela de camellones y en la parcela de control (Pampa). En la época de las mediciones, el cultivo tenía sobre ambos sitios una altura media de $0,5 \mathrm{~m}$ con un LAI alrededor de 2 . Durante los dos años, no hubo efecto de sequía perceptible sobre la parcela de la Pampa debido a una estación de lluvia relativamente abundante. Se colocó una estación meteorológica Campbell Scientific en el medio de cada sitio (camellones y parcela de control) para medir la temperatura del aire $\left(T_{a}\right)$, la humedad del aire $\left(h_{a}\right)$ y la radiación solar a una altura de $2 \mathrm{~m}$ sobre el nivel del suelo. Se usaron sensores de humedad relativa Vaisala HMP35C-L. Las diferencias en los valores registrados en ambos sitios siendo muy pequeñas, se utilizaron sistemáticamente, como referencias climáticas, los promedios de los dos valores de $T_{a} \mathrm{y} h_{a}$. La velocidad del viento $\left(u_{a}\right)$ a la altura de referencia se midió solamente el segundo año sobre el sitio principal (camellones) con un anemómetro Victor (tipo A 100R). Ambos años, se midieron las temperaturas del aire dentro del cultivo $\left(T_{a l 0}\right)$ a una altura de $10 \mathrm{~cm}$ en cada sitio, cada vez con dos replicas, utilizando sondas de temperatura de tipo Campbell Scientific 107B. El segundo año, se midió también la temperatura del agua $\left(T_{w 10}\right)$ por medio de dos de estos sensores colocados $10 \mathrm{~cm}$ bajo la superficie del agua. Los datos estaban registrados cada minuto y promediados sobre periodos de 15 minutos por medio de dos "data loggers" (Campbell Scientific CR10). No se midió ninguna radiación nocturna, pero según du Portal (1993) el déficit radiativo promedio durante noches de heladas en el altiplano avecina generalmente los $-75 \mathrm{~W} \mathrm{~m}^{-2}$.

\section{2. Resultados experimentales}

Las figuras 2a y 2b representan dos series temporales, obtenidas en 1994 y 1995, de la temperatura mínima diaria del cultivo de papa $\left(T_{a l 0}\right)$ medida en los camellones y en la Pampa (de hecho la temperatura del aire a una altura de $10 \mathrm{~cm}$ ). En 1994, la serie evoluciona aproximadamente entre $0^{\circ} \mathrm{C}$ y $-10^{\circ} \mathrm{C}$ : la temperatura mínima en la Pampa es casi siempre inferior de 1-2 grados a la misma temperatura medida en los camellones. El efecto de mitigación es evidente pero no sobrepasa los dos grados. En la serie de 1995, con temperaturas menos bajas (entre $0^{\circ} \mathrm{C} \mathrm{y}-4^{\circ} \mathrm{C}$ ), el efecto permanece pero es menos pronunciado, del orden de $1^{\circ} \mathrm{C}$. Estos datos concuerdan con los resultados obtenidos en la región altiplánica por Churra et al. (1992), Duran (1992) y Sánchez de Losada et al. (1998). La figura 3 muestra, para una noche característica en 1995, la variación temporal de la temperatura del cultivo $\left(T_{a 10}\right)$ medida en los campos levantados 

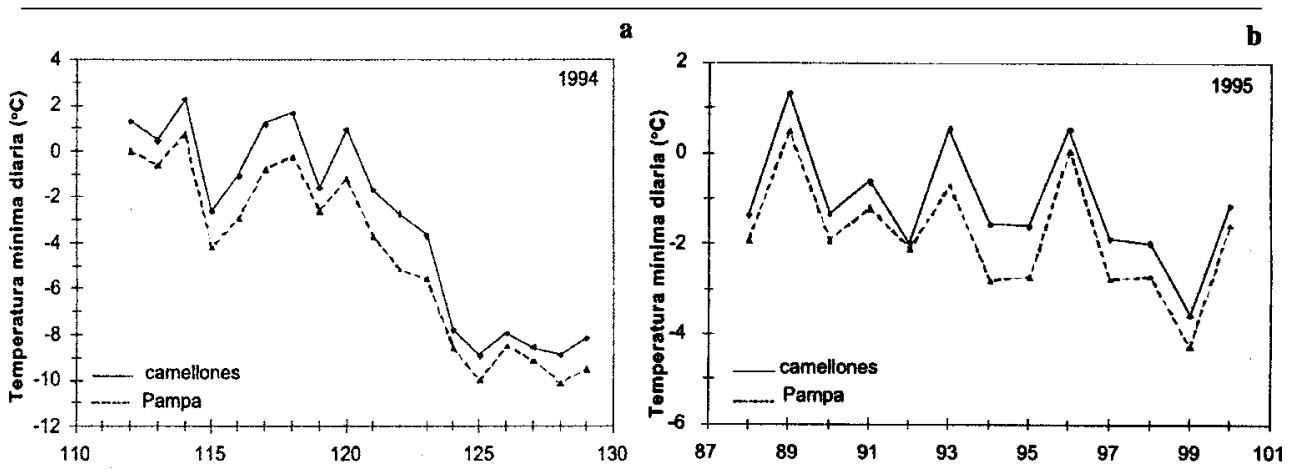

Fig. 2 - Comparación de la temperatura mínima diaria del cultivo $\left(T_{a 10}\right)$ en los camellones y en la Pampa:

a) serie temporal en 1994 (23 de abril - 10 de mayo); b) serie temporal en 1995 (30 de marzo - 11 de abril).

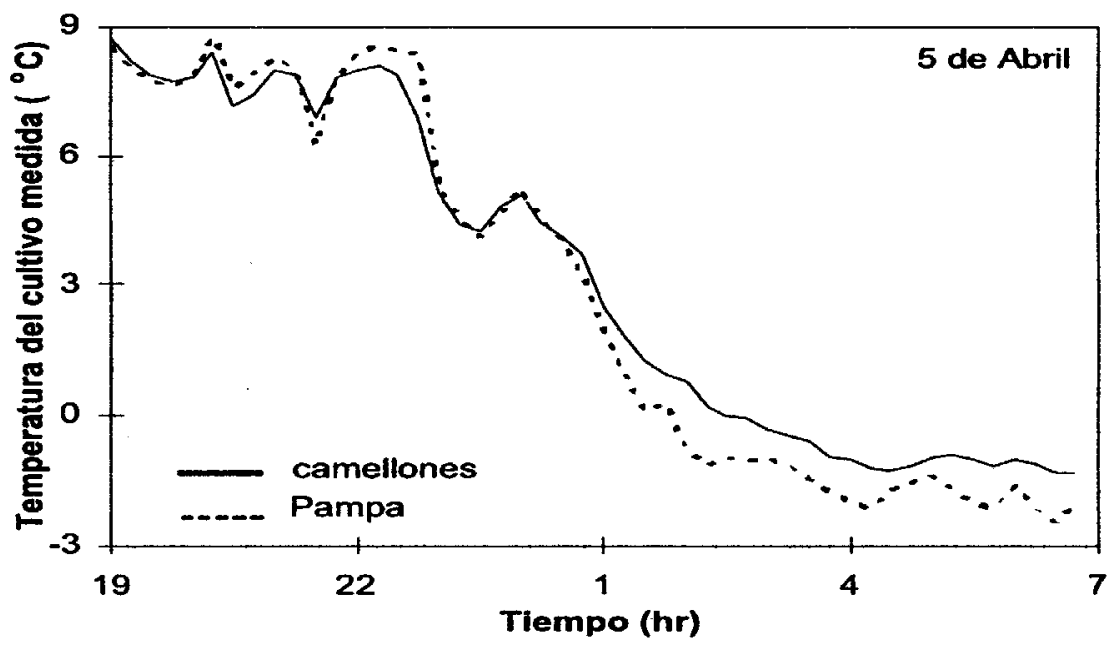

Fig. 3 - Para un día típico (5 de abril de 1995), variación nocturna de la temperatura del cultivo $\left(T_{a 10}\right)$ medida en el sistema de camellones comparada con la misma temperatura medida en la parcela de control (Pampa).

y la misma temperatura medida en la parcela de control. Durante la segunda mitad de la noche, la temperatura del cultivo se encuentra sistemáticamente más alta en el sistema de camellones, resaltando claramente así su papel de mitigación de heladas. En las figuras $4 \mathrm{a}$ y $4 \mathrm{~b}$ se ve claramente que durante toda la noche la temperatura del agua está mucho más alta que la temperatura del aire, sea al nivel de referencia climática o al nivel del cultivo con una diferencia del orden de $10{ }^{\circ} \mathrm{C}$. Existe por consiguiente una transferencia de calor en el aire del agua de los canales hacia el cultivo. Sánchez de Lozada et al. (1998) demonstraron experimentalmente que la transferencia de calor ocurre esencialmente en el aire y no en el suelo: el flujo de calor en el suelo, de los canales hacia las plataformas, no es suficiente como para contribuir de manera significante al efecto de mitigación de heladas. 

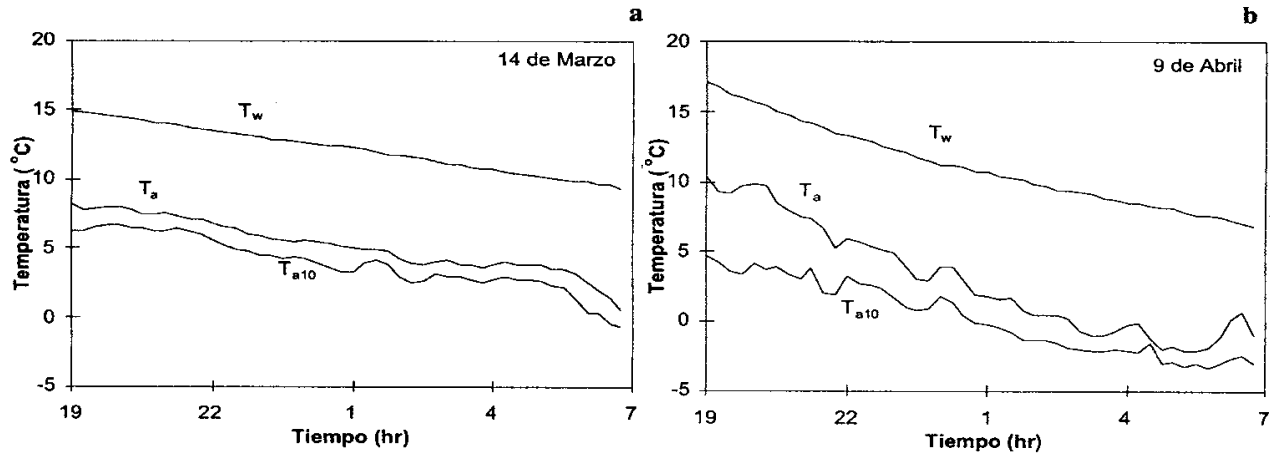

Fig. 4 - Variación nocturna de la temperatura del cultivo $\left(T_{a 10}\right)$, del agua $\left(T_{w}\right)$ y del aire $\left(T_{a}\right)$ en el sistema de camellones durante dos noches características:

a) 14 de marzo de 1995; b) 9 de abril de 1995.

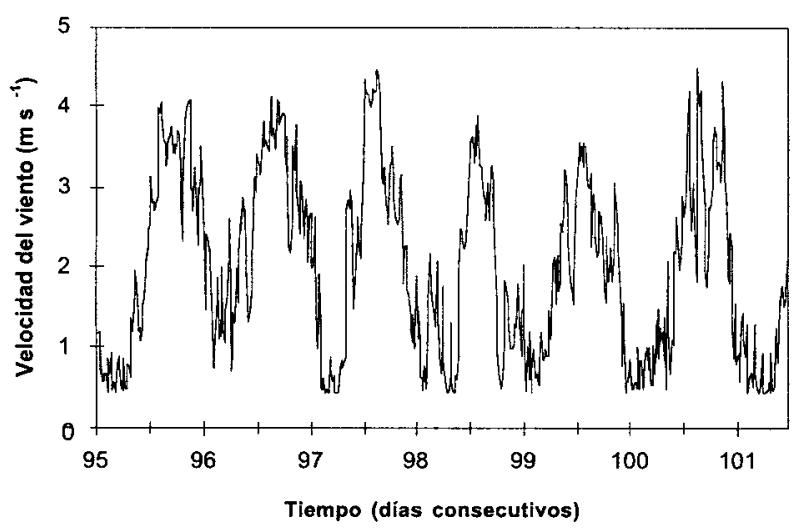

Fig. 5 - Variación temporal de la velocidad del viento a $2 \mathrm{~m}$ para una serie de días consecutivos (6 de abril - 12 de abril de 1995): sobre el eje $x$, la marca de cada dia corresponde al inicio del dia (o h) y la marca menor corresponde a mediodia (12 h).

Fig. 6 - Esquema teórico de las transferencias de energía en un sistema de

La figura 5 muestra cómo el viento evoluciona entre el día y la noche. La velocidad del viento es generalmente mucho más alta de día que de noche con un valor mínimo inferior a $1 \mathrm{~m} \mathrm{~s}^{-1}$ al final de la noche, cuando suelen ocurrir las heladas radiativas. El hecho que la velocidad del viento sea muy baja en la segunda parte de la noche contribuye a la ocurrencia de heladas, porque un viento fuerte tiende a favorecer la transferencia de calor del canal y del aire hacia el cultivo más frío. Cabe subrayar también que la evaporación del agua de los canales tiende a aumentar la humedad del aire y favorecer así la mitigación de heladas por el calor liberado en el proceso de condensación, sea por depósito de rocío sobre el suelo o las plantas o por la formación de niebla. Sin embargo las mediciones de humedad disponibles en el experimento (a la altura de $2 \mathrm{~m}$ ) no permiten poner en evidencia este fenómeno. 


\section{MODELO MICROMETEOROLÓGICO}

Presentamos en esta sección un modelo que describe las transferencias de calor y de vapor de agua dentro de los camellones de patrón lineal. Se utiliza como base el modelo de interacción vegetación-atmósfera de dos capas diseñado por Shuttleworth \& Wallace (1985) y completado por Choudhury \& Monteith (1988) y Shuttleworth \& Gurney (1990). La capa "sustrato" representa el canal lleno de agua y la capa "vegetación" representa el cultivo que se desarrolla sobre la plataforma. La figura 6 muestra un esquema que visualiza las transferencias en el sistema. La mayoría de las ecuaciones utilizadas provienen de este modelo básico donde las transferencias convectivas se describen utilizando la teoría de la difusión turbulenta ( $K$-theory), aún cuando el transporte turbulento dentro de la cubierta vegetal no es un proceso estricto de difusión (McNaughton \& van den Hurk, 1995). Tenemos que subrayar, también, que el modelo básico de Shuttleworth-Wallace no se adapta bien al sistema de camellones si el nivel del agua dentro de los canales está demasiado bajo. Cuando es el caso, se debe tener en cuenta de una cierta manera la diferencia de nivel entre las dos superficies (suelo y agua). Este punto se discute en el anexo 2.

El modelo con todos sus detalles matemáticos ha sido ya publicado en un artículo en inglés (Lhomme \& Vacher, 2002). Consecuentemente, damos aquí solamente las ecuaciones básicas que permiten entender la estructura del modelo. Las personas interesadas en la parte de modelación podrán reportarse al artículo citado para más detalles.

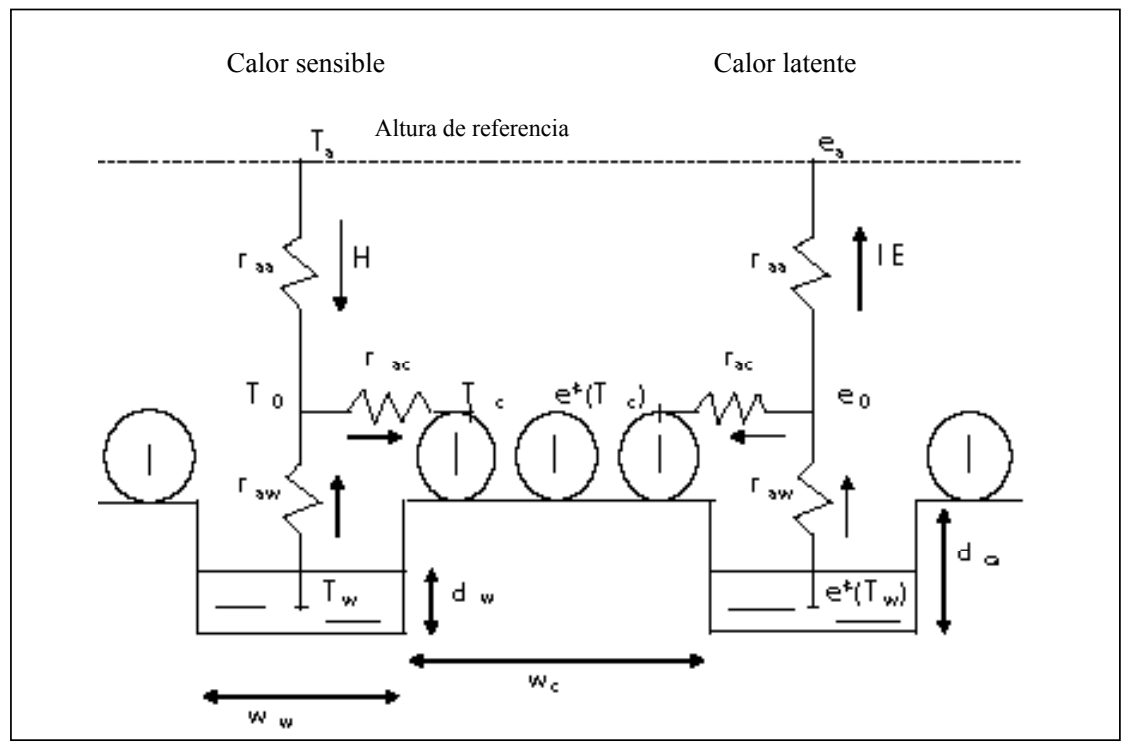

camellones. 


\section{1. Ecuaciones básicas} manera

El balance de energía de la capa de vegetación se escribe de la siguiente

$$
A_{c}=R_{n, c^{*}}-G_{c}=H_{c} \quad\left(+\lambda E_{c}\right)
$$

$A_{c}$ es la energía disponible del cultivo, $R_{n, c}$ es la radiación neta, $G_{c}$ el flujo de conduccíon del calor en el suelo, $H_{c}$ el flujo de calor sensible, $\lambda E_{c}$ el flujo de calor latente (de cambio de estado). Se supone que la vegetación no evapora durante la noche, lo que significa que $\lambda E_{c}=0$. Pero, cuando la temperatura del cultivo $T_{c}$ se vuelve más baja que la temperatura del punto de rocío del aire en la cubierta vegetal, puede ocurrir un depósito de rocío o escarcha. En este caso, hay que tomar en cuenta el flujo adicional de calor latente. Una ecuación similar se escribe para la capa de sustrato (agua de los canales) con el índice $w$ (water), pero con un término adicional de evaporación nocturna. Al anochecer, el agua libre que llena los canales está generalmente mucho más caliente que el suelo y su temperatura disminuye lentamente en el transcurso de la noche como consecuencia de las pérdidas radiativas, conductivas y convectivas. En este caso, además del balance de radiación y del flujo conductivo de calor $G_{w}$ (que circula del canal hacia el suelo), la energía disponible del agua $\left(A_{w}\right)$ debe involucrar la variación temporal de la cantidad de calor $C_{w}$ almacenada en el agua. Entonces, el balance de energía se escribe

$$
A_{w^{*}}=R_{n, w^{\prime}}-G_{w^{*}}-\frac{d C_{w^{\prime}}}{d t}=H_{w^{*}}+\lambda E_{\mathrm{w}^{\prime}}
$$

Podemos escribir también dos ecuaciones de conservación, que especifican que el flujo total que entra o sale del sistema es la suma de las contribuciones de cada capa. Para el calor sensible y el calor latente tenemos respectivamente

$$
H=H_{c}+H_{w} \quad \lambda E=\lambda E_{w} \quad\left(+\lambda E_{c}\right)
$$

Estas ecuaciones son detalladas en el anexo 1, los flujos convectivos siendo escritos en función de las temperaturas, presiones de vapor y resistencias siguiendo el esquema de la figura 6.

Las energías disponibles del cultivo y del agua deben ser ponderadas por el área relativa de cada superficie (Lhomme \& Chehbouni, 1999). Para un sistema de campos "infinitamente" largos con plataformas de ancho $w_{c}$, bordeadas con canales rectangulares de ancho $w_{w}$, el área relativa del cultivo y del agua son respectivamente $w_{c} /\left(w_{c}+w_{w}\right)$ y $w_{w} /\left(w_{c}+w_{w}\right)$. Durante la noche, suponiendo que el flujo de calor del suelo es una fracción $c(c>0)$ de la radiación neta, la energía disponible del cultivo se escribe (con $T_{c}$ expresado en Kelvin)

$$
A_{c}=\left[w_{c} /\left(w_{c}+w_{w}\right)\right](1-c) \varepsilon_{c}\left(R_{a}-\sigma T_{c}^{4}\right)
$$

donde $R_{a}$ es la radiación incidente de onda larga, $\varepsilon_{c}$ es la emisividad del cultivo y $\sigma$ es la constante de Stefan-Boltzmann. La energía disponible del agua $A_{w}$ se detalla de la siguiente manera

$$
A_{w}=\left[w_{w} /\left(w_{c}+w_{w}\right)\right] \varepsilon_{w}\left(R_{a}-\sigma T_{w}^{4}\right)-G_{w}-\rho_{w} c_{w} V_{w}\left(d T_{w} / d t\right)
$$


donde $\varepsilon_{w}$ es la emisividad del agua, $d T_{w} / d t$ es la variación de la temperatura del agua por unidad de tiempo, $V_{w}$ es el volumen de agua por unidad de superficie, $\rho_{w}$ es la densidad del agua y $c_{w}$ es el calor específico del agua. Para un sistema de plataformas "infinitamente" largas bordeadas con canales rectangulares, $V_{w}$ se escribe $V_{w}=w_{w} d_{w}$ $/\left(w_{c}+w_{w}\right)$, donde $d_{w}$ representa la profundidad del agua en los canales. En cuanto al flujo de calor transferido por conducción, puede escribirse

$$
G_{w}=h_{w} a_{w}\left(T_{w}-T_{s}\right)
$$

donde $T_{s}$ es la temperatura del suelo a una profundidad de referencia, suficientemente profunda para que sea considerada como constante y independiente de las fluctuaciones diarias. La temperatura $T_{s}$ será considerada como igual a la temperatura media registrada a la altura de referencia $\left(z_{r}\right)$ encima de la cubierta vegetal, una aproximación respaldada por el análisis formal de la conducción térmica en el suelo (Monteith \& Unsworth, 1990). El coeficiente de intercambio $h_{w}$ (por unidad de área de canal en contacto con el agua libre) será obtenido empiricamente a partir de los datos experimentales (véase la sección "Calibración y validación del modelo"). El parámetro $a_{w}$ representa el área relativa del canal en contacto con el agua (y por consiguiente, que intercambia calor con el suelo), expresada por $a_{w}=\left(w_{w}+2 d_{w}\right) /\left(w_{c}+w_{w}\right)$.

El objetivo general del modelo es de poder simular durante la noche la variación temporal de la temperatura del cultivo $T_{c}(t)$ y del agua $T_{w}(t)$ a partir de la variación temporal de los datos meteorológicos [i.e., $\left.T_{a}(t), e_{a}(t), u_{a}(t)\right]$ registrados a nivel de la caseta meteorológica y conociendo la temperatura inicial del agua $T_{w}(0)$. Se supone que la variación correspondiente de la radiación incidente de onda larga $R_{a}(t)$ está conocida, sea calculada a partir de los datos de entrada o directamente medida. $R_{a}(t)$ es de hecho raramente medida per se. En el modelo se la calcula por medio de la fórmula propuesta por Brutsaert (1982) en función de la temperatura $T_{a}$ y humedad $e_{a}$ del aire. Esta fórmula, con bases físicas, se escribe

$$
R_{a}=\varepsilon_{a} \sigma T_{a}^{4} \quad \varepsilon_{a}=\mu\left(e_{a} / T_{a}\right)^{1 / 7}
$$

El parámetro $\mu$ es un coeficiente que varía con la nubosidad y que será ajustado a las condiciones experimentales (véase "Calibración del modelo"). Tiene un valor de 1,24 bajo condiciones de cielo claro y de atmósfera estándar $\left(e_{a}\right.$ siendo expresado en $\mathrm{hPa}$ y $T_{a}$ en $\left.\mathrm{K}\right)$.

Elmodelo presentado se articula de la siguiente manera: un sistema de 4 ecuaciones con cuatro incognitas (entre ellas $T_{c}$ y $T_{w}$ ) se resuelve en cada paso de tiempo $i$. Con el fin de simplificar la resolución del sistema de ecuaciones, $T_{c}^{4}$ y $T_{w}^{4}$ en las Ecs. (4) y (5) pueden ser linearizadas. Entonces, el sistema se vuelve linear y tiene una solución algébrica simple explicitada en Lhomme \& Vacher (2002). Si un depósito de rocío ocurre, la resolución del conjunto de ecuaciones no es tan evidente, porque el sistema ya no es linear. Entonces un método numérico se vuelve necesario; está también descrito en Lhomme \& Vacher (2002).

La temperatura del cultivo en los camellones $\left(T_{c}\right)$, calculada de la manera 
descrita arriba, será comparada con la obtenida en una parcela de control en la Pampa $\left(T_{c, p}\right)$, donde se encuentra el mismo cultivo pero sin canales de agua. En este caso, la temperatura $T_{c, p}$ es la solución de la siguiente ecuación del balance de energía (detallada en el anexo 1)

$$
A_{p}=R_{n, p}-G_{p}=H_{p} \quad\left(+\lambda E_{p}\right)
$$

con los mismos símbolos que arriba, el índice $p$ referiéndose al cultivo en la Pampa. Cuando ocurre un depósito de rocío, el término adicional de calor latente $\lambda E_{p}$ debe tomarse en cuenta.

\section{2. Calibración y validación del modelo}

La comparación entre modelo y experimento se hizo con los datos de 1995, ya que en 1994 no se había registrado la temperatura del agua. De todos los datos obtenidos se utilizaron solamente las siete noches con las temperaturas mínimas más bajas, la primera siendo utilizada para calibrar el modelo. Las noches seleccionadas aparecen en la Tabla 1. Dado que experimentos anteriores habían mostrado que el cociente $c$ del flujo de calor del suelo sobre la radiación neta del cultivo era alrededor de 0,50 durante la noche, este valor, entonces, se utilizó sistemáticamente en el modelo. En las condiciones del altiplano, alrededor de $4000 \mathrm{~m}$ sobre el nivel del mar, la presión atmosférica $(P)$ es muy baja, cerca de $650 \mathrm{hPa}$. Eso significa que la constante psicrométrica $\gamma=P c_{p} /(0.622 \lambda)$, que aparece en las ecuaciones del modelo, es mucho más baja que el valor común de $67 \mathrm{~Pa}^{\circ} \mathrm{C}^{-1}$. El valor correcto, utilizado aquí, es $\gamma=43$ $\mathrm{Pa}{ }^{\circ} \mathrm{C}^{-1}$. El coeficiente $\mu$, que define la radiación incidente de onda larga $R_{a}$ (Ec. (7)), tiene un valor que depende esencialmente de la nubosidad del cielo. Dado que no se hizó ninguna medición directa de $R_{a}$ durante el experimento (tampoco estimaciones de

Tabla 1 - Temperaturas del aire y del agua para cada día seleccionado de 1995. $T_{a}$ es la temperatura del aire a la altura de referencia con tres diferentes índices $(x$ : máxima diaria, $n$ : mínima diaria, $m$ : media diaria); $T_{w}(0)$ es la temperatura del agua al inicio de la noche $(19: 00 \mathrm{~h})$; el coeficiente $\mu$ define la radiación incidente de onda larga [Ec. (7)].

\begin{tabular}{|ccccccc|}
\hline Fecha & DDS $^{*}$ & $\mathrm{~T}_{\mathrm{a}, \mathrm{x}}\left({ }^{\circ} \mathrm{C}\right)$ & $\mathrm{T}_{\mathrm{a}, \mathrm{n}}\left({ }^{\circ} \mathrm{C}\right)$ & $\mathrm{T}_{\mathrm{a}, \mathrm{m}}\left({ }^{\circ} \mathrm{C}\right)$ & $\mathrm{T}_{\mathrm{w}}(0)\left({ }^{\circ} \mathrm{C}\right)$ & $\mu$ \\
4 de marzo & 108 & 16,2 & 2,4 & 9,3 & 19,3 & 1,10 \\
5 de marzo & 109 & 16,7 & 0,0 & 8,4 & 19,4 & 1,03 \\
14 de marzo & 118 & 15,0 & 4,5 & 9,8 & 14,9 & 1,33 \\
2 de abril & 137 & 17,0 & 0,6 & 8,8 & 15,5 & 1,26 \\
5 de abril & 140 & 17,7 & $-0,6$ & 8,6 & 15,6 & 1,24 \\
9 de abril & 144 & 18,0 & $-0,9$ & 8,6 & 17,1 & 1,10 \\
10 de abril & 145 & 18,7 & $-2,5$ & 8,1 & 15,8 & 1,22 \\
\hline
\end{tabular}

"día después de la siembra 
la nubosidad), se ajustó un valor medio de $\mu$ a los datos experimentales medidos sobre el sitio de la Pampa. Para cada noche de medición, el valor medio de $\mu$ retenido es el que minimiza la RMSE (Root Mean Square Error) entre la temperatura del cultivo $\left(T_{c, p}\right)$ calculada por la Ec. $(8)$ y la temperatura del cultivo medida $\left(T_{a 10}\right)$. Los valores obtenidos aparecen en la Tabla 1 (más nublado está el cielo, más alto está el coeficiente $\mu)$. Otro parámetro calibrado es el coeficiente de intercambio de calor $h_{w}$ en la Ec. (6). Usando los datos de la primera noche (4 de marzo), la RMSE entre la temperatura del agua calculada por el modelo $\left(T_{w}\right)$ y la temperatura del agua medida $10 \mathrm{~cm}$ debajo de la superficie $\left(T_{w 10}\right)$ ha sido minimizada; el valor obtenido es $h_{w}=35 \mathrm{~W} \mathrm{~m}^{-2} \mathrm{~K}^{-1}$. Se dan más detalles al respecto en Lhomme \& Vacher (2002).

En la comparación entre el modelo y el experimento tenemos que subrayar que la temperatura del cultivo calculada $\left(T_{c}\right)$ representa la temperatura media de las hojas, mientras que la temperatura "del cultivo" medida $\left(T_{a 10}\right)$ es en realidad la temperatura del aire a una altura de $10 \mathrm{~cm}$ dentro de la cubierta. Las dos temperaturas no son exactamente iguales y alguna discrepancia puede existir entre las dos. La figura 7 muestra para dos noches características ( 5 de marzo y 2 de abril) la variación de las temperaturas del cultivo y del agua, medida y simulada por el modelo. Si la temperatura del cultivo $\left(T_{c}\right)$ aparece correctamente simulada, la temperatura del agua $\left(T_{w}\right)$ lo es con una menor precisión. Está globalmente sobre-estimada el 5 de marzo, mientras que está sub-estimada el 2 de abril. La discrepancia podría explicarse por una mala estimación del coeficiente de intercambio $h_{w}$ en la Ec. (6), que ha sido empiricamente calibrado a partir de los datos del 4 de marzo. El coeficiente $h_{w}$ tiene un impacto importante sobre el valor de $T_{w}$, ya que determina la cantidad de calor perdida por el agua libre hacia el suelo. La figura 8 compara la temperatura del cultivo calculada a partir del modelo con la medida, para cada paso de tiempo $(15 \mathrm{mn})$ de las seis noches de mediciones. La línea de regresión forzada por el origen es $T_{c}=1,07 T_{a 10}$ con $R^{2}=0,95$ y la RMSE (Root Mean Square Error) es igual a $0,78^{\circ} \mathrm{C}$. Son resultados que podemos considerar como satisfactorios. Hay que subrayar que una relación muy estrecha existe entre la temperatura del cultivo y la temperatura del aire $\left(T_{a 10}=0,94 T_{a}-1,67 \operatorname{con} R^{2}=0,92\right)$, lo que significa que la mayor parte de la variación de la temperatura del cultivo se debe
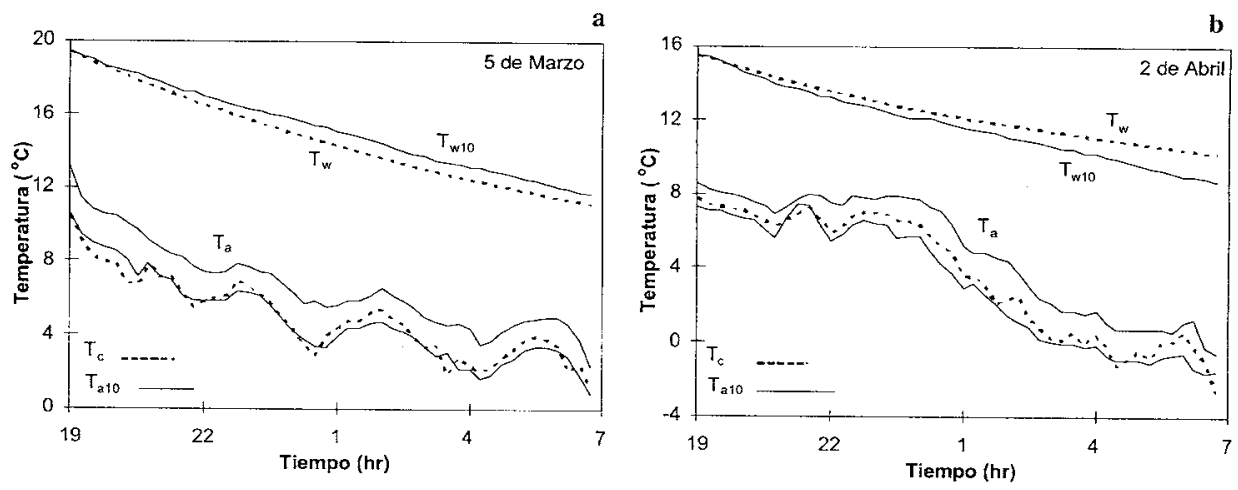

Fig. 7 - Variación temporal de las temperaturas del cultivo y del agua durante dos noches, medida y simulada por el modelo:

a) 5 de marzo de 1995; b) 2 de abril de 1995. 


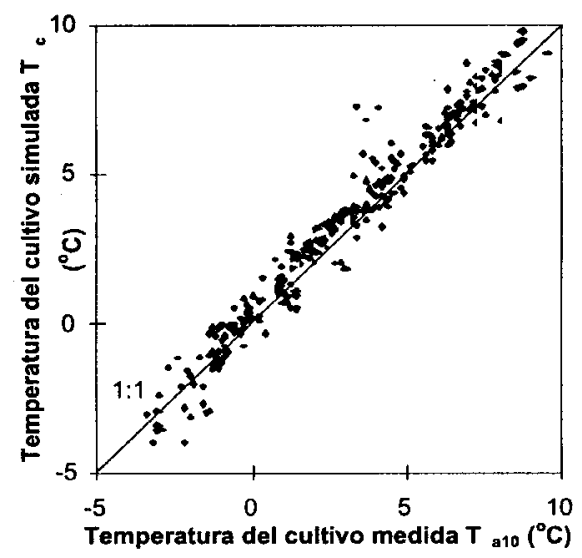

Fig. 8 - Temperatura del cultivo simulada versus temperatura del cultivo medida, para cada paso de tiempo (15 min) de los seis días seleccionados de 1995.

a la variación de la temperatura del aire.

\section{PREDICCIÓN DEL MODELO}

En esta sección el modelo calibrado y comprobado se utiliza a fines predictivas. Variando las características geométricas de los camellones y del cultivo se evalúa el impacto correspondiente sobre la temperatura mínima alcanzada durante la noche.

\section{1. Impacto de las características geométricas de los camellones}

La geometría del sistema de camellones es sintetizada esencialmente en cuatro parámetros: el ancho del canal $\left(w_{w}\right)$, la profundidad del canal $\left(d_{c a}\right)$, la profundidad del agua dentro del canal $\left(d_{w}\right)$ y el ancho de las plataformas $\left(w_{c}\right)$. Utilizando los datos meteorológicos registrados en el transcurso de una noche dada, se corre el modelo variando sucesivamente el valor de un parámetro de la geometría, manteniendo todos los demás parámetros iguales. En cada simulación, el modelo suministra una temperatura mínima del cultivo $T_{c, n}$ (la temperatura del cultivo más baja alcanzada durante la noche). Las figuras 9a, 9b, 9c y 9d representan las variaciones de $T_{c, n}$ en función de las características geométricas de los camellones (ancho de la plataforma, ancho del canal, profundidad del agua y profundidad del canal). Los valores básicos utilizados en el proceso de simulación son casi similares a los del experimento: ancho de la plataforma $w_{c}=4 \mathrm{~m}$, ancho del canal $w_{w}=2 \mathrm{~m}$, profundidad del canal $d_{c a}=1,5 \mathrm{~m}$, profundidad del agua $d_{w}=1 \mathrm{~m}$, altura del cultivo $h=0,5 \mathrm{~m}$, índice de área foliar $L_{0 c}=2$. Las simulaciones se realizaron utilizando los datos meteorológicos registrados durante dos noches características (el 5 de marzo y 2 de abril). La temperatura del suelo $T_{s}$ a una profundidad de referencia (considerada igual a la temperatura media del aire $T_{a, m}^{s}$ ) y la temperatura inicial del agua $T_{w}(0)$ fueron utilizadas tales como se midieron estos 
días (Tabla 1). Cabe señalar que las tendencias generales presentendas en la figura 9 no cambian si se usan los datos meteorológicos de cualquier otra noche, lo que justifica a posteriori el procedimiento utilizado.

La figura 9a muestra la variación de $T_{c, n}$ en función del ancho de la plataforma. Todas las demás condiciones siendo iguales, $T_{c, n}$ disminuye de $1-2^{\circ} \mathrm{C}$ cuando el ancho de la plataforma aumenta de $0,5 \mathrm{~m}$ a $10 \mathrm{~m}$ con un efecto marcado de $0,5 \mathrm{a} 2 \mathrm{~m}$. En la figura $9 \mathrm{~b}$, se representa $T_{c, n}$ en función del ancho del canal $w_{w}$. Cuando $w_{w}$ aumenta de $0,5 \mathrm{~m}$ a $4 \mathrm{~m}, T_{c, n}$ puede subir de $2^{\circ} \mathrm{C}$ ( 2 de abril). Como se podía preverlo intuitivamente, estos resultados resaltan la importancia que tiene en el proceso de mitigación de heladas una gran proporción de canales con respecto a las plataformas (sin olvidar no obstante la pérdida consecuente de superficie cultivada). Sin embargo, estos resultados simulados por el modelo deben considerarse con cierto cuidado. Hay que señalar que cuando la plataforma es demasiada ancha con respecto al canal, o viceversa, la validez del modelo bi-capa para representar el sistema de campos levantados podría questionarse. Eso significa prácticamente que los resultados obtenidos para valores altos de $w_{c} \mathrm{o} w_{w}$ (digamos, mayores de 6-8 m) podrían ser erróneos.

La figura 9c muestra la variación de la temperatura mínima del cultivo $T_{c, n}$ en función de la profundidad del agua $\left(d_{w}\right): T_{c, n}$ lógicamente se incrementa con la profundidad del agua, pero la tasa de incremento (pendiente de la curva) no es constante y muy leve hasta $1 \mathrm{~m}$; es más grande para grandes valores de $d_{w}$. Eso significa prácticamente que para aumentar el efecto de mitigación de heladas, es recomendable tener canales profundos y llenos de agua. La figura 9d muestra la variación de $T_{c n}$ en función de la profundidad del canal $\left(d_{c a}\right)$ para una profundidad constante de agua $\left(d_{w}=1 \mathrm{~m}\right)$. Revela que un canal más profundo, con la misma cantidad de agua, tiene un efecto negativo sobre la mitigación de heladas. Es la resistencia adicional $r_{a w, e x}$ [proporcional a $\left(d_{c a}\right.$ $-d_{w}$ ), Ec. (2.4)] que explica porque $T_{c, n}$ disminuye cuando $d_{c a}$ aumenta, ya que esta resistencia tiende a limitar la transferencia de calor del agua hacia el cultivo. El costo marginal de un canal más profundo, sin embargo, es realmente significativo solamente para valores bajos de $\left(d_{c a}-d_{w}\right): T_{c, n}$ disminuye mucho más cuando $\left(d_{c a}-d_{w}\right)$ varía de 0 a $0,5 \mathrm{~m}$ que cuando varía de $0,5 \mathrm{~m}$ a $2 \mathrm{~m}$.

\section{2. Impacto de las características del cultivo y de las condiciones meteorológicas}

Un procedimiento similar al precedente es utilizado para estudiar el impacto de las características físicas del cultivo (altura, LAI) sobre la mitigación de heladas. Utilizando los datos meteorológicos de varias noches, se ha corrido el modelo sucesivamente variando solamente uno de estos dos parámetros, todos los demás parámetros siendo iguales. La figura 10a muestra que el efecto de la altura de las plantas sobre la temperatura mínima del cultivo es globalmente positivo. Cuando la altura del cultivo varía de 0,1 a $2 \mathrm{~m}$, la temperatura mínima aumenta de 1 a casi $3{ }^{\circ} \mathrm{C}$ según los días. El LAI tiene también un impacto claramente positivo sobre la mitigación de heladas (Fig. 10b), sobre todo entre 0 y 2 . Cuando el LAI varía de 0 a 2, la temperatura mínima del cultivo aumenta de aproximadamente $3^{\circ} \mathrm{C}$, variación muy significativa. Se puede explicar este efecto global por el hecho de que el aumento de la altura e índice foliar del cultivo tiende a disminuir las resistencias aerodinámicas encima y dentro de la cubierta vegetal y eso 

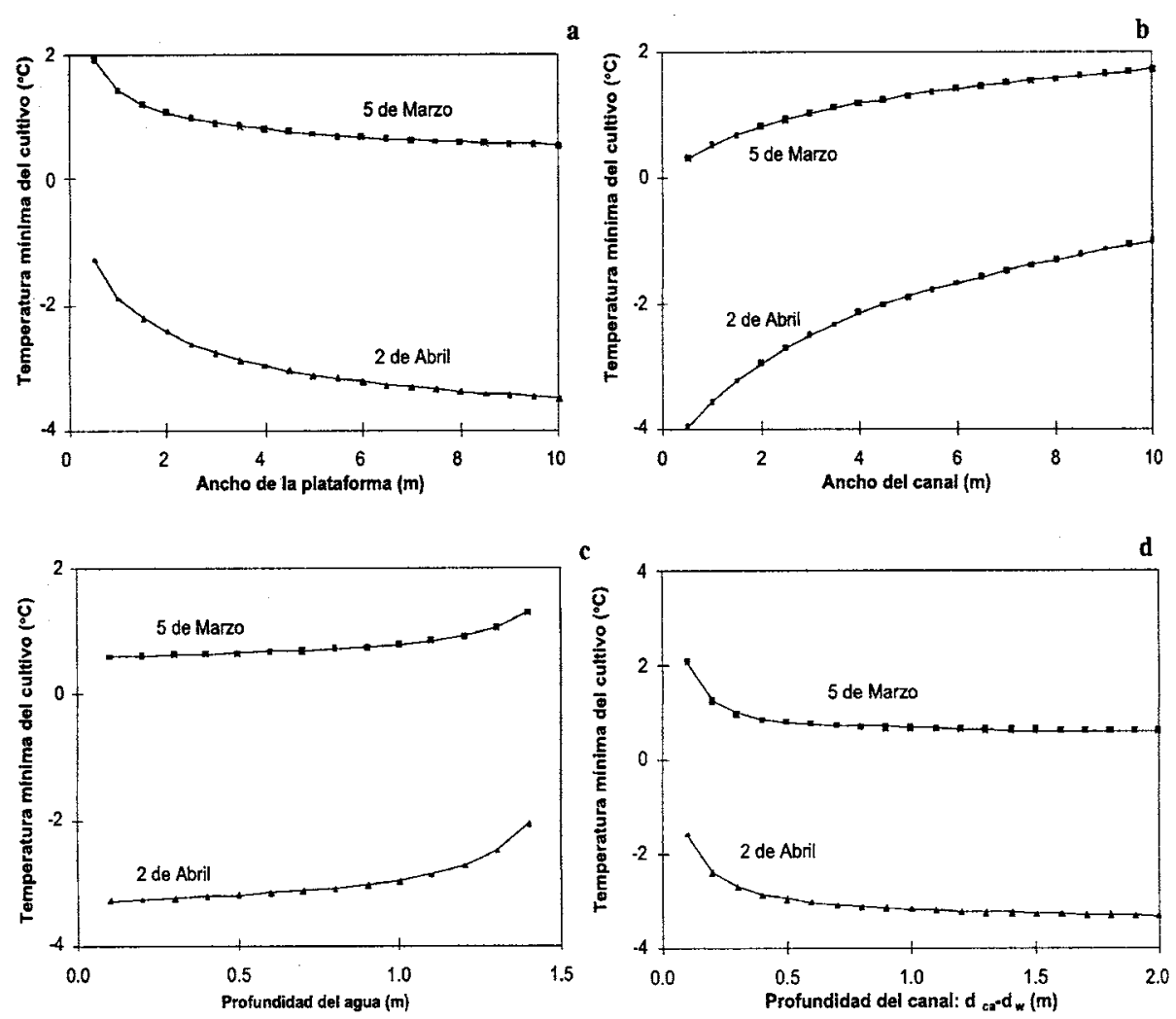

Fig. 9 - Temperatura mínima del cultivo alcanzada durante dos noches (5 de marzo, 2 de abril de 1995) en función de las características geométricas de los camellones, todas las demás condiciones siendo iguales $\left(w_{c}=4 \mathrm{~m}, w_{w}=2 \mathrm{~m}, d_{c a}=1,5\right.$ $\left.\mathrm{m}, d_{w}=1 \mathrm{~m}, z_{h}=0,5 \mathrm{~m}, L_{0 c}=2\right)$ :

a) ancho de la plataforma $\left(w_{c}\right)$; b) ancho del canal $\left(w_{w}\right)$; c) profundidad del agua $\left.\left(d_{w}\right) ; \mathrm{d}\right)$ profundidad del canal $\left(d_{c a}-d_{w}\right)$.

favorece la transferencia de calor del aire y del agua hacia el cultivo.

Se ha examinado también el impacto de las condiciones meteorológicas (velocidad del viento, humedad del aire) utilizando un procedimiento similar. Se ha corrido el modelo con los datos registrados durante varias noches, variando sucesivamente uno de los dos parámetros, pero manteniendo constante su valor durante toda la noche. La velocidad del viento es un factor positivo en la mitigación de heladas dado que favorece la transferencia de calor del canal y del aire hacia el cultivo. La figura 10c muestra que la temperatura mínima del cultivo aumenta de aproximadamente $2{ }^{\circ} \mathrm{C}$ cuando la velocidad constante del viento pasa de 0,5 a $3 \mathrm{~m} \mathrm{~s}^{-1}$ (variaciones frecuentes en el altiplano), todas las demás condiciones siendo iguales. Sin embargo, el beneficio marginal disminuye con el aumento de velocidad. La humedad del aire tiene también un impacto positivo sobre la mitigación de heladas, lo que muestra la figura 10d. Cuando 

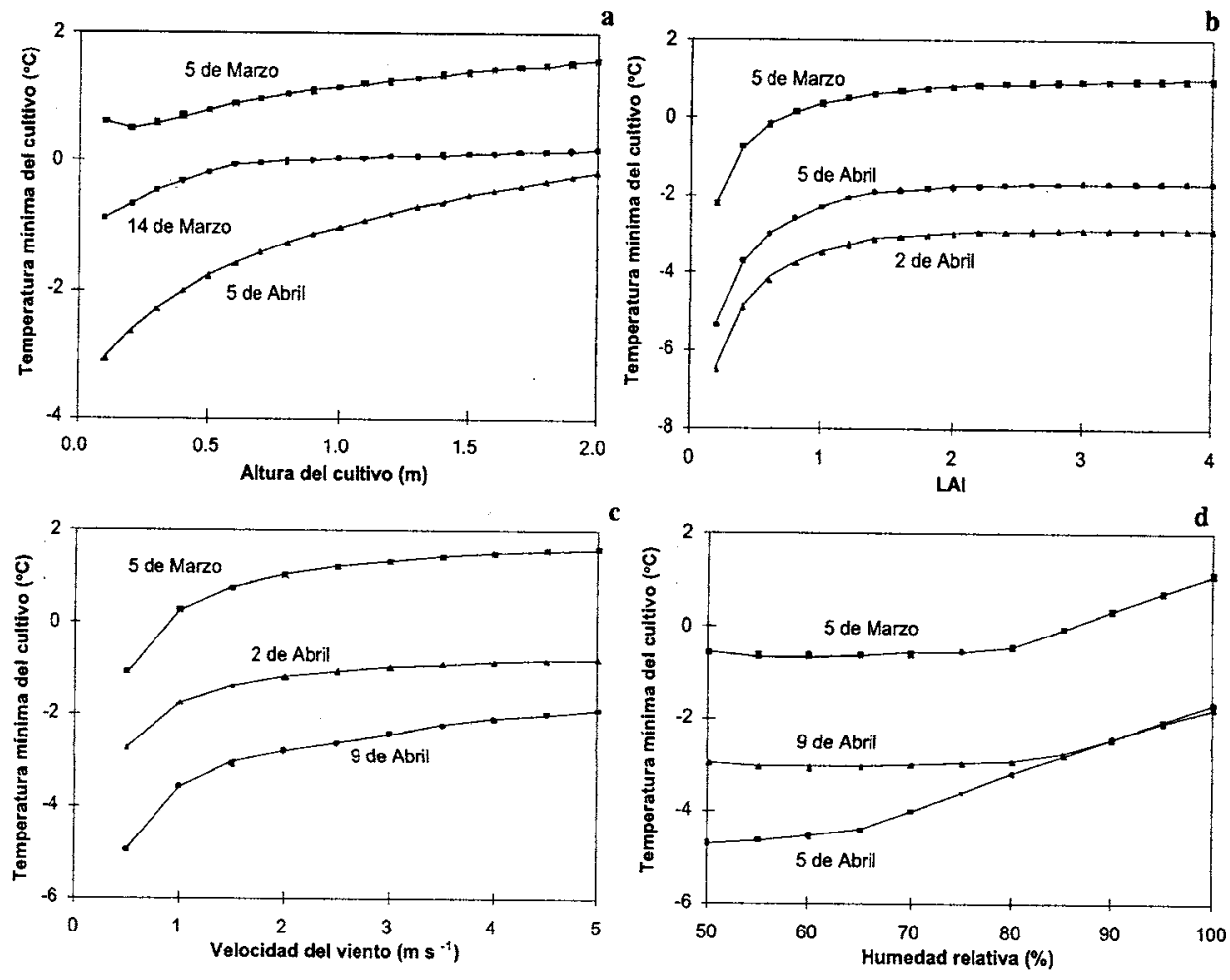

Fig. 10 - Temperatura mínima del cultivo alcanzada durante la noche en función de las características del cultivo y de las condiciones meteorológicas, todas las demás condiciones siendo iguales $\left(w_{c}=4 \mathrm{~m}, w_{w}=2 \mathrm{~m}, d_{c a}=1,5 \mathrm{~m}, d_{w}=1 \mathrm{~m}, z_{h}=0,5 \mathrm{~m}, L_{0 c}=\right.$ 2) :

a) altura del cultivo; b) LAI del cultivo; c) velocidad del viento a una altura de referencia de $\mathbf{2} \mathbf{m}$ (mantenida constante); d) humedad del aire a una altura de

la humedad relativa $h_{a}$ (mantenida constante durante la noche) aumenta de 50 a 100 $\%$, la temperatura mínima del cultivo puede incrementar de $1-3{ }^{\circ} \mathrm{C}$ según los días. Este efecto es obviamente relacionado con una mayor condensación del vapor de agua sobre las hojas y un balance radiativo menos negativo.

\section{CONCLUSIÓN}

Se ha podido comprobar experimentalmente que la dinámica nocturna del calor en el sistema de camellones del altiplano andino permite mitigar las heladas nocturnas. El efecto de mitigación es frecuentemente inferior a $2^{\circ} \mathrm{C}$. Sin embargo, es suficiente para tener una consecuencia nítida sobre la disminución de los riesgos de heladas. Un modelo de tipo mecanístico, basado sobre una descripción bi-capa de la interacción superficie-atmósfera (un sustrato constituido por el agua de los canales y una capa de vegetación) ha sido desarrollado para representar y analizar este sistema. El modelo 
calcula simultáneamente la variación nocturna de la temperatura del cultivo y del agua a partir de los datos meteorológicos a un nivel de referencia y de la temperatura inicial del agua. El efecto conjunto del calor sensible transferido del agua de los canales hacia el cultivo y del agua condensada sobre las hojas (como consecuencia de una humedad del aire más alta por la evaporación de los canales), llega a tener un impacto sustancial de mitigación sobre las heladas radiativas nocturnas, impacto que depende de las características geométricas del sistema. El modelo muestra que un canal más ancho o una plataforma más estrecha tienen un impacto positivo sobre la temperatura mínima del cultivo alcanzada durante la noche. Incrementar la profundidad del agua mejora también la mitigación de las heladas pero levemente. A la inversa, un canal más profundo (con la misma profundidad de agua) tiene un impacto negativo, lo que confirma, en este caso, que el drenaje de aire frío hacia los canales no es el mecanismo físico pertinente responsable del efecto de mitigación. En cuanto a las características del cultivo, altura y LAI, ambas tienen un impacto positivo cuando se incrementan, facilitando de una cierta manera la transferencia del calor de los canales y del aire hacia el cultivo. Un aumento de la velocidad del viento o de la humedad del aire igualmente incrementa el efecto de mitigación.

Ahora que antiguas superficies de waru waru están rehabilitatas sobre el altiplano andino y que se están construyendo nuevas, el modelo de base física presentado en este estudio debería revelarse útil en sus diseños prácticos, ya que permite estimar para condiciones meteorológicas dadas el efecto de la geometría de los camellones sobre la temperatura mínima del cultivo.

\section{Referencias citadas}

BOUYSSE, T., MORLON, P., MOURGUIART, P. \& WIRMANN, D., 1992 - Agricultura, sociedad y medio natural en la cuenca del Titicaca: Tres mil años de relaciones. In: Actas del VII Congreso Internacional sobre Cultivos Andinos, (Vacher J. J. and Morales eds.): 383-388; La Paz.

BRUTSAERT, W., 1982 -Evaporation into the Atmosphere, 299p.; Dordrecht, The Netherlands: Kluwer Academic Publishers.

CANAHUA MURILLO, A. \& LARICO MAMANI, L., 1992 - Manual técnico de waru waru para profesionales y técnicos, 305p.; Puno, Perú: Programa Interinstitucional de Waru waru (PIWA).

CARI CHOQUEHUANCA, A. \& CAMACHO ARCE, C., 1992 - Principios técnicos para la reconstrucción y producción agrícola en waru waru. I. Suelos y agua, 199p.; Programa Interinstitucional de Waru waru (PIWA); Puno.

CHOUDHURY, B. J. \& MONTEITH, J. L., 1988 - A four-layer model for the heat budget of homogeneous land surfaces. Quarterly Journal of the Royal Meteorological Society, 114: 373-398.

CHURA, E., CARI, A., CAÑAHUA, A., VACHER, J. J. \& DÍAZ, C., 1992 - Variación de la temperatura mínima en agrosistemas de camellones. In: II Producción agrícola: 51-69; Puno, Perú: Programa Interinstitucional de Waru Waru (PIWA).

DIAZ, C. \& VELÁZQUEZ, E., 1992 - Inventario de Infraestructuras Agrícolas Andinas en Puno- Perú. In: Avances de Investigación sobre la tecnología de Waru-Waru: 17-38.

DU PORTAL, D., 1993 - Études des gelées sur l'Altiplano Bolivien. Mémoire de DAA, ENSA. M, Montpellier, France. 58p. 
DURAN, C., 1992 -Habilitación de terrenos no aptos para la agricultura mediante la construcción de camellones en la región de Caquiaviria. Tésis Ing. Agrónomo, UMSA, La Paz, Bolivia. 251p.

ERICKSON, C.L., 2000 - An artificial landscape-fishery in the Bolivian Amazon . Nature, 408: $190-193$.

ERICKSON, C.L., 1988 - Raised field agriculture in the Lake Titicaca bassin: putting ancient Andean agriculture back to work. Expedition, 30(3): 8-16.

ERICKSON, C.L., 1986a - Waru-waru: una tecnología agrícola del Altiplano pre-hispánico. In: Andenes y Camellones en el Perú Andino (C. de la Torre \& M. Burga Eds.): 59-84; Lima: CONCYTEC.

ERICKSON, C.L., 1986b - Agricultura en camellones en la cuenca del Lago Titicaca: aspectos técnicos y su futuro. In: Andenes y Camellones en el Perú Andino (C. de la Torre \& M. Burga Eds.): 331-350M Lima: CONCYTEC.

FLORES OCHOA, J.A. \& PAZ FLORES, P., 1986 - La agricultura en lagunas (qocha). In: Andenes y Camellones en el Perú Andino (C. de la Torre \& M. Burga Eds.); Lima: CONCYTEC.

GRACE, B., 1988 - El clima del Altiplano, Departamento de Puno, Perú. 182p.; Convenio Perú-Canada. Estación Experimental Illpa-Puno, Perú.

KOLATA, A. L. \& ORTLOFF, C., 1989 - Thermal analysis of Tiwanaku raised fields in the Lake Titicaca basin of Bolivia. Journal of Archaeological Science, 16: 233-263.

LHOMME, J.-P. \& CHEHBOUNI, A., 1999-Comments on dual-source vegetation-atmosphere transfer models. Agricultural and Forest Meteorology, 94: 269-273.

LHOMME, J.-P. \& VACHER, J. J., 2002-Modelling nocturnal heat dynamics and frost mitigation in Andean raised field systems. Agricultural and Forest Meteorology, 112: 179-193.

MCNAUGHTON, K. G. \& VAN DEN HURK, B. J. J. M., 1995 - A 'Lagrangian' revision of the resistors in the two-layer model for calculating the energy budget of a plant canopy. Boundary Layer Meteorol., 74: 261-288.

MONTEITH, J.L. \& UNSWORTH, M. H., 1990 - Principles of Environmental Physics, 247p.; Londres: E. Arnold.

MORLON, P., 1992 - Réduction des risques climatiques par les aménagements : l'exemple des gelées sur l'Altiplano. In: Comprendre l'Agriculture Paysanne dans les Andes Centrales (P. Morlon Ed.), 265-277; Paris: INRA.

MORRIS, A., 1999 - The agricultural base of the pre-Incan Andean cicvilizations. The Geographical Journal, 165: 286-295.

MURRA, J.V., 1975 - Formaciones Económicas y Políticas del Mundo Andino, 339p.; Lima: Instituto de Estudios Peruanos.

RILEY, T. M. \& FREIMUTH, G., 1979 - Field systems and frost drainage in the prehistoric agriculture of the upper Great Lakes. American Antiquity, 44: 271-285.

SÁNCHEZ DE LOZADA, D., BAVEYE, P. \& RIHA, S., 1998 - Heat and moisture dynamics in raised field systems of the lake Titicaca region (Bolivia). Agricultural and Forest Meteorology, 92: 251-265.

SHUTTLEWORTH, W. J. \& WALLACE, J. S., 1985 - Evaporation from sparse crops - an energy combination theory. Quarterly Journal of the Royal Meteorological Society, 111: $839-855$.

SHUTTLEWORTH, W. J. \& GURNEY, R. J., 1990 - The theoretical relationship between foliage temperature and canopy resistance in sparse crops. Quarterly Journal of the Royal Meteorological Society, 116: 497-519.

SMITH, C. T., DENEVAN, W. M. \& HAMILTON, P., 1968 -Ancient ridged fields in the region of Lake Titicaca. The Geographical Journal, 134: 353-367.

VACHER, J. J., 1998 - Responses of two mains Andean crops, quinoa (Chenopodium quinoa Willd) and papa amarga (Solanum juzepczukii Buk.) to drought on the Bolivian Altiplano: Significance of local adaptation. Agriculture, Ecosystems and Environment, 68: 99108. 


\section{Anexo 1 - Detalle de las ecuaciones básicas}

Las ecuaciones (1) y (2) se detallan de la siguiente manera

$$
\begin{aligned}
& A_{c}=\rho c_{p}\left(T_{c}-T_{0}\right) / r_{a c}\left[+\left(\rho c_{p} / \gamma\right)\left(e^{*}\left(T_{c}\right)-e_{0}\right) / r_{a c}\right] \\
& A_{w}=\left(\rho c_{p} / \gamma\right)\left[e^{*}\left(T_{w}\right)-e_{0}\right] / r_{a w}+\rho c_{p}\left(T_{w}-T_{0}\right) / r_{a w}
\end{aligned}
$$

$T_{0} \mathrm{y} e_{0}$ son respectivamente la temperatura del aire y la presión de vapor del aire a la altura de fuente de la cubierta vegetal (canopy source height: $\left.d+z_{0}\right) \cdot e^{*}(T)$ es la presión de vapor de saturación a la temperatura $T . r_{a w}$ es la resistencia aerodinámica entre la superficie del agua y la altura de fuente de la cubierta vegetal. $r_{a c}$ es la resistencia global de capa límite del follaje. $\gamma$ es la constante psicrométrica, $\rho$ la densidad del aire y $c_{p}$ el calor específico del aire a presión constante.

Las ecuaciones (3) se escriben respectivamente

$$
\begin{gathered}
\left(T_{a}-T_{0}\right) / r_{a a}=\left(T_{0}-T_{c}\right) / r_{a c}+\left(T_{0}-T_{w}\right) / r_{a w} \\
\left(e_{a}-e_{0}\right) / r_{a a}=\left[e_{0}-e^{*}\left(T_{w}\right)\right] / r_{a w} \quad\left[+\left(e_{0}-e^{*}\left(T_{c}\right)\right) / r_{a c}\right]
\end{gathered}
$$

$r_{a a}$ es la resistencia aerodinámica entre la altura de fuente $\left(d+z_{0}\right)$ y la altura de referencia $\left(z_{r}\right)$, donde se miden los datos meteorológicos $\left(T_{a} \mathrm{y} e_{a}\right)$.

La ecuación (8) que da la temperatura del cultivo en la Pampa $T_{c, p}$ se escribe

$$
(1-c) \varepsilon_{c}\left[R_{a}-\sigma T_{c, p}^{4}\right]=\rho c_{p}\left(T_{c, p}-T_{a}\right) / r_{a}
$$

donde $r_{a}$ es la resistencia aerodinámica de la parcela de control. Las formulaciones de las resistencias que componen el modelo se detallan en el anexo 2.

\section{Anexo 2 - Especificación de las resistencias en el aire}

Se supone que la resistencia aerodinámica encima de la cubierta vegetal $r_{a a}$ es la misma para el calor sensible y el vapor de agua. Considerando también que los gradientes de temperatura en el aire están relativamente pequeños durante la noche, se calcula $r_{a q}$ sin tomar en cuenta las funcciones de corrección de estabilidad para los perfiles de viento y temperatura, como sigue

$$
r_{a a}=\frac{1}{k^{2} u_{a}} \ln ^{2}\left(\frac{z_{r}-d}{z_{0}}\right)
$$


$u_{a}$ es la velocidad del viento en la altura de referencia $z_{r}$ y $k$ es la constante de von Karman. La altura de desplazamiento del plano zero $d$ y la longitud de rugosidad para la cantidad de moviniento $z_{0}$ se determinan siguiendo Choudhury \& Monteith (1988). Las formulas correspondientes están en Lhomme \& Vacher (2002). La resistencia aerodinámica de la parcela de control se calcula de una manera similar.

Las resistencias del aire dentro de la cubierta vegetal se calculan utilizando las formulaciones propuestas por Choudhury \& Monteith (1988). La resistencia global de capa límite de la cubierta se obtiene integrando la conductancia de capa límite foliar sobre la altura de la cubierta, suponiendo que el índice de área foliar está uniformemente distribuido sobre esta altura

$$
r_{a c}=\frac{\alpha_{u}\left[w_{l} / u\left(z_{h}\right)\right]^{1 / 2}}{4 \alpha_{0} L_{0}\left[1-\exp \left(-\alpha_{w} / 2\right)\right]}
$$

$w_{l}$ es la anchura de la hoja; $u\left(z_{h}\right)$ es la velocidad del viento a la altura de la cubierta vegetal, calculada suponiendo un perfil logarítmico; $\alpha_{0}$ y $\alpha_{w}$ son dos constantes iguales respectivamente a $0,005 \mathrm{~m} \mathrm{~s}^{-1 / 2}$ y 2,5 (sin dimensión). La resistencia aerodinámica entre el sustrato (la superficie del agua) y la altura de fuente de la cubierta vegetal $\left(d+z_{0}\right)$ se define como la integral del inverso de la difusividad turbulenta sobre el intervalo de altura $\left[z_{0 s}, d+z_{0}\right]$ (Choudhury \& Monteith, 1988)

$$
r_{a w}=\frac{z_{h} \exp \left(\alpha_{w}\right)}{\alpha_{w} K\left(z_{h}\right)}\left\{\exp \left[-\alpha_{w} z_{0 s} / z_{h}\right]-\exp \left[-\alpha_{w}\left(d+z_{0}\right) / z_{h}\right]\right\}
$$

donde $K\left(z_{h}\right)$ es el valor de la difusividad turbulenta a la altura $z_{h}$ de la cubierta vegetal. La altura de rugosidad del sustrato $z_{0_{s}}$ es calculada como el valor medio de las alturas de rugosidad del suelo y del agua libre.

En la formulación de la resistencia aerodinámica dentro de la cubierta [Ec. (2.3)], se supone implicitamente que el agua y el suelo están al mismo nivel. Sin embargo, los canales generalmente no están llenos de agua, y por consiguiente, el nivel del agua está más bajo. El calor y el vapor de agua transferidos del agua hacia el aire tienen que atravesar más distancia, que se debe tomar en cuenta por medio de una resistencia adicional sumada a la resistencia aerodinámica $r_{a w}$. Por falta de una teoría adecuada, se utiliza un enfoque puramente empírico para parametrizar esta resistencia. Se supone que es proporcional a la diferencia de nivel entre el suelo y el agua (profundidad del canal $\left(d_{c a}\right)$ menos la profundidad del agua $\left.\left(d_{w}\right)\right)$ y inversamente proporcional a la velocidad del viento $\left(u_{a}\right)$ y a la anchura del canal $\left(w_{w}\right)$

$$
r_{a w, e x}=\beta\left(d_{c a}-d_{w}\right) / w_{w} u_{a}
$$

En efecto, es lógico suponer que más bajo está el nivel de agua, más grande es la resistencia adicional (opuesta a la transferencia de calor y de vapor de agua), y que más ancho es el canal, más pequeña es la resistencia, el viento penetrando más facilmente dentro del canal. $\beta$ es un coeficiente de proporcionalidad sin dimensión, que fue ajustado usando los datos experimentales de la primera noche (4 de Marzo) de las siete noches seleccionadas. La RMSE entre la temperatura del cultivo calculada por el modelo $\left(T_{c}\right)$ y la temperatura medida del cultivo $\left(T_{a 10}\right)$ ha sido minimizada. El valor obtenido es de aproximadamente $4,8 \times 10^{3}$ (sin dimensión), lo que hace que $r_{a w}$ y $r_{a w, e x}$ son más o menos del mismo orden de magnitud. 


\section{Lista de símbolos}

$a_{w} \quad$ área relativa de los canales en contacto con el agua (sin dimensión)

$A_{c} \quad$ energía disponible del cultivo $\left(\mathrm{W} \mathrm{m}^{-2}\right)$

$A_{w} \quad$ energía disponible del agua $\left(\mathrm{W} \mathrm{m}^{-2}\right)$

c razón del flujo de calor del suelo a la radiación neta del cultivo $(0,50)$

$c_{p} \quad$ calor específico del aire a presión constante $\left(\mathrm{J} \mathrm{kg}^{-1} \mathrm{~K}^{-1}\right)$

$c_{w} \quad$ calor específico del agua (4 $\left.200 \mathrm{~J} \mathrm{~kg}^{-1} \mathrm{~K}^{-1}\right)$

$d \quad$ altura de desplazamiento del plano zero $(\mathrm{m})$

$d_{c a} \quad$ profundidad de los canales (m)

$d_{w} \quad$ profundidad del agua dentro de los canales (m)

$e_{a} \quad$ presión de vapor de agua del aire a la altura de referencia $(\mathrm{Pa})$

$e_{0} \quad$ presión de vapor de agua del aire a la altura fuente de la cubierta $(\mathrm{Pa})$

$e^{*}(T) \quad$ presión de vapor saturado a la temperatura $T(\mathrm{~Pa})$

$G_{w} \quad$ flujo de calor por conducción entre el agua libre y el suelo $\left(\mathrm{W} \mathrm{m}^{-2}\right)$

$h_{a} \quad$ humedad relativa a la altura de referencia (\%)

$h_{w} \quad$ coeficiente de intercambio de calor entre el agua libre y el suelo ( $\left.\mathrm{W} \mathrm{m}^{-2} \mathrm{~K}^{-1}\right)$

$H \quad$ flujo de calor sensible $\left(\mathrm{W} \mathrm{m}^{-2}\right)$

$k \quad$ constante de von Karman (0.4)

$K \quad$ coeficiente de difusión turbulenta $\left(\mathrm{m}^{2} \mathrm{~s}^{-1}\right)$

$L_{0} \quad$ LAI (Leaf Area Index): índice de área foliar por unidad de área total $\left(\mathrm{m}^{2} \mathrm{~m}^{-2}\right)$

$L_{0 c} \quad$ índice de área foliar por unidad de área de plataforma (Clumped Leaf Area Index) $\left(\mathrm{m}^{2} \mathrm{~m}^{-2}\right)$

$r_{a} \quad$ resistencia aerodinámica de la parcela de control (Pampa) $\left(\mathrm{s} \mathrm{m}^{-1}\right)$

$r_{a a} \quad$ resistencia aerodinámica de los camellones (entre altura de fuente y altura

$\mathrm{de} \quad$ referencia) $\left(\mathrm{s} \mathrm{m}^{-1}\right)$

$r_{a c} \quad$ resistencia global de capa límite de la vegetación $\left(\mathrm{s} \mathrm{m}^{-1}\right)$

$r_{a w} \quad$ resistencia del aire entre la superficie del agua y la altura de fuente $\left(\mathrm{s} \mathrm{m}^{-1}\right)$

$r_{a w, e x} \quad$ resistencia adicional a sumar a $r_{a w}\left(\mathrm{~s} \mathrm{~m}^{-1}\right)$

$R_{a} \quad$ radiación incidente de onda larga $\left(\mathrm{W} \mathrm{m}^{-2}\right)$

$T_{a} \quad$ temperatura del aire a la altura de referencia $\left({ }^{\circ} \mathrm{C}\right)$

$T_{a 10} \quad$ temperatura del cultivo medida:

temperatura del aire a una altura de $10 \mathrm{~cm}$ dentro de la cubierta vegetal $\left({ }^{\circ} \mathrm{C}\right)$

$T_{c} \quad$ temperatura del cultivo simulada por el modelo $\left({ }^{\circ} \mathrm{C}\right)$

$T_{c, n} \quad$ temperatura mínima del cultivo alcanzada durante la noche $\left({ }^{\circ} \mathrm{C}\right)$ 
$T_{s} \quad$ temperatura del suelo a una profundidad de referencia $\left({ }^{\circ} \mathrm{C}\right)$

$T_{w} \quad$ temperatura del agua de los canales $\left({ }^{\circ} \mathrm{C}\right)$

$T_{0} \quad$ temperatura del aire a la altura de fuente $=$

Temperatura aerodinámica de superficie $\left({ }^{\circ} \mathrm{C}\right)$

$u_{a} \quad$ velocidad del viento a la altura de referencia $\left(\mathrm{m} \mathrm{s}^{-1}\right)$

$V_{w} \quad$ volumén de agua por unidad de área total $\left(\mathrm{m}^{3} \mathrm{~m}^{-2}\right)$

$w_{c} \quad$ ancho de la plataforma $(\mathrm{m})$

$w_{w} \quad$ ancho del canal (m)

$z_{0} \quad$ longitud de rugosidad de la cubierta vegetal (m)

$z_{h} \quad$ altura de la cubierta vegetal (m)

$z_{r} \quad$ altura de referencia sobre el nivel del suelo $(2 \mathrm{~m})$

\section{Símbolos griegos:}

$\beta \quad$ coeficiente en la Ec. $(2,4)$ que define $r_{a w, e x}$ (sin dimensión)

$\gamma \quad$ constante psicrométrica $\left(\mathrm{Pa} \mathrm{K}^{-1}\right)$

$\varepsilon_{c} \quad$ emisividad de la vegetación $(0,97)$

$\varepsilon_{w} \quad$ emisividad del agua $(0,97)$

$\lambda \quad$ calor latente de vaporización $\left(\mathrm{J} \mathrm{kg}^{-1}\right)$

$\lambda \mathrm{E} \quad$ flujo de calor latente $\left(\mathrm{W} \mathrm{m}^{-2}\right)$

$\mu \quad$ coeficiente empírico en la Ec. (7) que define $R_{a}$ (sin dimensión)

$\rho \quad$ densidad del aire $\left(\mathrm{kg} \mathrm{m}^{-3}\right)$

$\rho_{w} \quad$ densidad del agua $\left(\mathrm{kg} \mathrm{m}^{-3}\right)$

$\sigma \quad$ constante de Stefan-Boltzmann $\left(5,67 \times 10^{-8} \mathrm{~W} \mathrm{~m}^{-2} \mathrm{~K}^{-4}\right)$ 


\section{Jean-Pierre Lavaud}

\section{La dictadura minada}

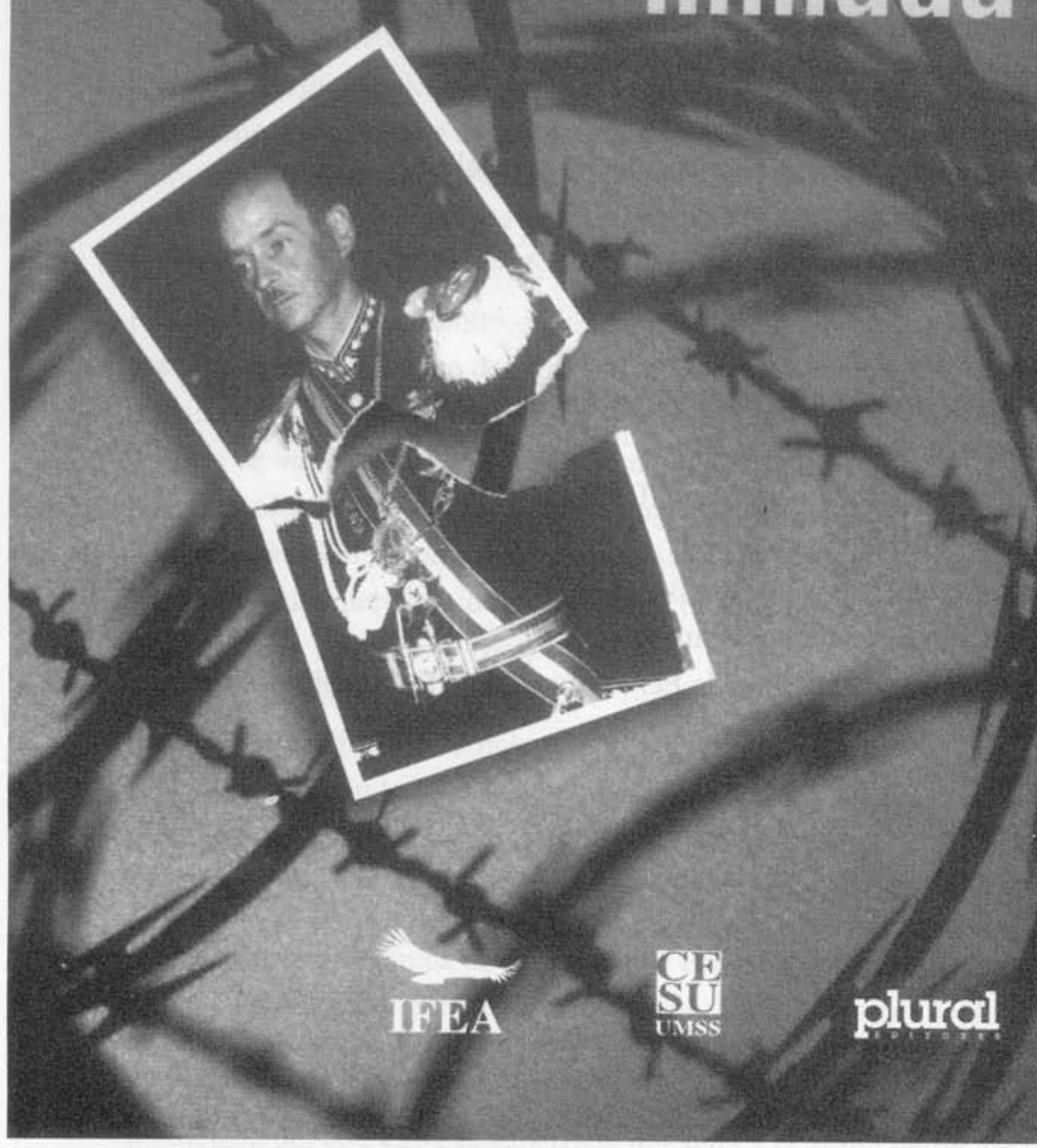

Institut Français d'Études Andines - CESU - PLURAL Editores

Pedidos: IFEA, Casilla 18-1217, Lima 18 - Perú, Tel. 4476070 Fax: 4457650 - E-mail: postmaster@ifea.org.pe Web: http://www.ifeanet.org 